\title{
Invasive Aspergillus tracheobronchitis in a patient with hairy cell leukemia and previous Plasmodium falciparum infection
}

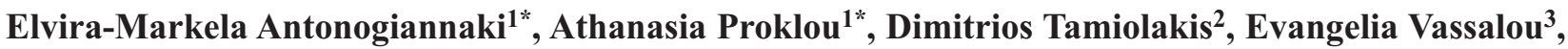 \\ Eumorfia Kondili ${ }^{1}$ \\ ${ }^{1}$ Department of Intensive Care Medicine, University Hospital of Heraklion, Crete; ${ }^{2}$ Department of Pathology-Cytopathology, \\ University Hospital of Heraklion, Crete; ${ }^{3}$ Department of Radiology, General Hospital of Sitia, Crete, Greece; *These authors \\ contributed equally
}

\begin{abstract}
Invasive Aspergillus tracheobronchitis is a relatively rare form of invasive pulmonary aspergillosis characterized by invasion of the tracheobronchial tree by Aspergillus spp. Invasive pulmonary aspergillosis is predominantly detected in severely immunocompromised patients. Notably however, pulmonary and tracheobronchial cases of invasive aspergillosis have also been reported, particularly in the context of severe malaria caused by Plasmodium falciparum. Herein, we present a case of invasive Aspergillus tracheobronchitis in a patient with hairy cell leukemia and previous Plasmodium falciparum infection.
\end{abstract}

\section{Introduction}

Invasive Aspergillus tracheobronchitis (IATB) is a unique, relatively rare variant of invasive pulmonary aspergillosis, in which

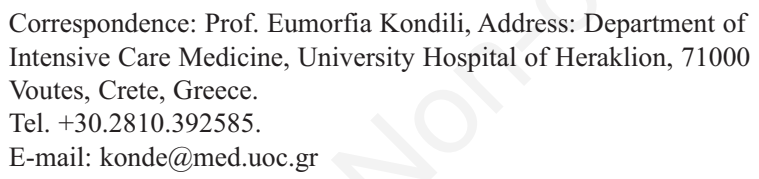

Key words: Invasive Aspergillus tracheobronchitis; malaria.

Contributions: All authors contributed to conception and design of the study case, interpretation of data, drafting the article or revising it critically for important intellectual content, and on final approval of the version to be published.

Conflict of interest: The authors have no conflict of interest to declare.

Received for publication: 25 February 2019

Accepted for publication: 18 April 2019.

(C) Copyright E.-M. Antonogiannaki et al., 2019

Licensee PAGEPress, Italy

Monaldi Archives for Chest Disease 2019; 89:1055

doi: 10.4081/monaldi.2019.1055

This article is distributed under the terms of the Creative Commons Attribution Noncommercial License (by-nc 4.0) which permits any noncommercial use, distribution, and reproduction in any medium, provided the original author(s) and source are credited.
Aspergillus infection is entirely or predominantly confined to the tracheobronchial tree. Three different types of IATB have been described based on bronchoscopic findings; obstructive, pseudomembranous, and ulcerative [1]. Obstructive IATB is characterized by thick mucus plugs, while pseudomembranous IATB is characterized by extensive involvement of the tracheobronchial tree with a membranous layer covering the mucosal surface, and ulcerative IATB is associated with limited involvement of the tracheobronchial tree with focal, ulcerative, or plaque-like processes. IATB most commonly occurs in immunocompromised patients, though cases of isolated tracheobronchial aspergillosis have also been reported in mildly immunocompromised subjects [2-3], and more rarely in immunocompetent patients after infection with immunomodulatory microorganisms such as Plasmodium falciparum. Malaria-induced immunosuppression is apparent late in the acute or even during the recovery phase of the disease, occasionally leading to opportunistic infections including invasive aspergillosis [4-5]. IATB is a rapidly progressive and potentially fatal infection. Comparatively high mortality rates have been reported in severely immunosuppressed hosts including patients with hematological malignancies and transplant recipients [6]. Herein, we report a case of obstructive IATB in a patient with hairy cell leukemia and recent infection caused by Plasmodium falciparum.

\section{Case Report}

A 50-year-old Caucasian male who had resided in Cameroon for the past year presented to the Emergency Department complaining of persistent high fever, chills, and excessive fatigue. His medical history included hairy cell leukemia since 2002, with episodes of relapse in 2010 and 2016 for which he received chemotherapy with purine analog drug and with the monoclonal antibody rituximab on addition, during his disease relapse. There was no evidence of disease activity since 2016. One month earlier he had been diagnosed with acute malaria due to Plasmodium falciparum infection in Cameroon, and despite receiving adequate therapy with atovaquone/artesunate, he remained febrile and neutropenic. On admission, physical examination revealed fever of $39.0^{\circ} \mathrm{C}$, sinus tachycardia, tachypnea, and splenomegaly. Blood-smear examination revealed leucopenia with a white blood cell (WBC) count of $200 / \mu \mathrm{L}$, mild anemia with hemoglobin of $7.2 \mathrm{~g} / \mathrm{dL}$, and thrombocytopenia with a platelet count of $7000 / \mu \mathrm{L}$. The differential WBC count revealed zero neutrophils, and severe lymphopenia $(100 / \mu \mathrm{L})$. Apart from a mild increase in lactate dehydrogenase $(202 \mathrm{mg} / \mathrm{dL})$, and moderate hyperbilirubinemia (total $/$ unconjugated $=4.25 \mathrm{mg} / \mathrm{dL}$ 
$/ 1.12 \mathrm{mg} / \mathrm{dL}$ ), other lab results were unremarkable. He was admitted to the Internal Medicine Department and received de novo therapy against Plasmodium with atovaquone/artesunate and artemether. Due to persistent pancytopenia a bone marrow biopsy was performed, revealing diffuse marrow infiltration $(85-90 \%)$ by small neoplastic mononuclear lymphoid cells with a characteristic "fried egg" appearance, findings that were consistent with hairy cell leukemia. He was transferred to the Intensive Care Unit (ICU) 4 days later due to acute respiratory failure, persistent high temperature, and severe pancytopenia, despite targeted therapy against Plasmodium falciparum. On ICU admission, chest high resolution computed tomography (HRCT) revealed extensive areas of consolidation bilaterally, surrounded by ground-glass opacity together with multiple randomly distributed parenchymal nodules circumscribed by a ground-glass attenuation halo. There were also bilateral pleural effusions, more pronounced on the right, and enlarged mediastinal lymph nodes (Figure 1). Differential diagnosis included fungal pulmonary infectious process -especially angioinvasive aspergillosisor pulmonary bacterial infection associated with immunosuppression. Treatment against Plasmodium falciparum infection was discontinued after three consecutive negative thick blood films for $P$. falciparum, and empirical therapy with broad spectrum antibiotics and antifungal therapy with amphotericin-B was initiated. On the following days he exhibited significant clinical deterioration, with progressive respiratory failure, persistent high temperature up to $39.5^{\circ} \mathrm{C}$, and pancytopenia.

A second chest CT with intravenous contrast administration revealed deterioration compared to the previous imaging findings. Observations included enlargement of the pulmonary nodules and further progression of the areas of consolidation, which were characterized by the presence of multiple round or lobulated low-density regions with peripheral enhancement as well as areas of parenchymal decreased attenuation and hypoenhancement, indicative of necrosis. Further progression of the bilateral pleural effusions and mediastinal lymphadenopathy were also evident. He had progressively present refractory hypoxemia and was intubated due to severe acute respiratory distress syndrome (ARDS). Following intubation, flexible bronchoscopy was performed revealing complete obstruction of the right main lower bronchus caused by thick mucus plugs with an intensely hemorrhagic appearance (Figure 2). Bronchoalveolar lavage (BAL) samples were obtained for microbiological and cytological examination. Microbiological examination was positive for Aspergillus spp . In addition for conventional processing, direct smears were prepared from the fresh BAL samples; slides were fixed immediately in $95 \%$ alcohol and stained using Papanicolaou's method [7]. Cytological examination of the BAL fluid revealed hyaline septate mycelium filaments of 2-4 $\mu \mathrm{m}$ in diameter with occasional branching at acute angles, findings consistent with Aspergillus spp. (Figure 3) [7]. The patient died 2 days later due to multiple organ failure and severe ARDS.

\section{Discussion}

Invasive pulmonary aspergillosis is a life-threatening opportunistic infection, and immunosuppressed patients are particularly susceptible to it [6]. Both the extent and the duration of neutropenia, mainly after chemotherapy, are the major determinants of the risk for invasive pulmonary aspergillosis. Other common risk factors are hematological malignancies, bone marrow transplantation (especially allogeneic), solid organ malignancies, solid organ transplantation, HIV infection splenomegaly, and corticosteroid or other immunosuppressive treatment.

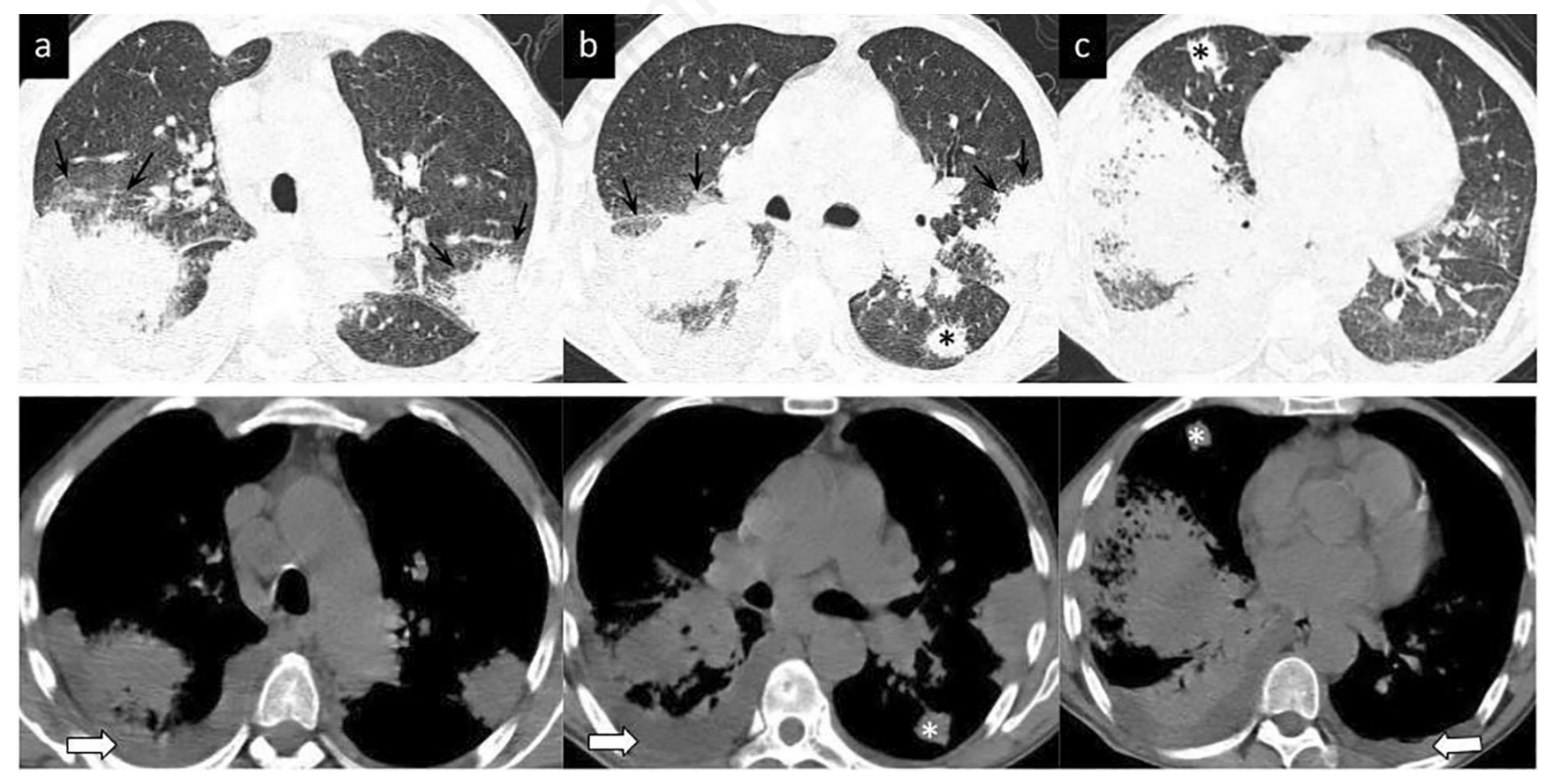

Figure 1. Chest high resolution computed tomography images through the upper (a) middle (b) and lower (c) lung fields. Upper row depicts images with "lung" and lower row with "mediastinal" window settings. Extensive areas of consolidation surrounded by groundglass opacity (arrows) as well as parenchymal nodules (asterisks) surrounded by a ground-glass attenuation halo ("halo sign") are demonstrated bilaterally. Note the presence of bilateral pleural effusions (open arrows), more pronounced on the right. 
IATB is a relatively rare form of invasive pulmonary aspergillosis in which Aspergillus infection is entirely or predominantly confined to the tracheobronchial tree Invasive pulmonary and tracheobronchial aspergillosis share common predisposing factors. Notably however, cases of isolated tracheobronchial aspergillosis have been reported in immunocompetent or mildly immunocompromised subjects [2-3] with conditions such as insulin-dependent diabetes, underlying autoimmune disorders, chronic pulmonary obstructive diseases, and tracheal structural abnormalities. In the majority of tracheobronchial infections Aspergillus fumigatus is identified as the causative species.

Based on bronchoscopic findings, three morphological variants of IATB have been described, obstructive tracheobronchitis, ulcerative tracheobronchitis, and pseudomembranous necrotizing bronchial aspergillosis [1]. The obstructive form is characterized by excessive mucus production, thick mucus plugs, and tracheal and/or bronchial inflammation. Ulcerative Aspergillus tracheobronchitis is characterized by focal, ulcerative, or plaque-like lesions of the tracheobronchial tree. Pseudomembranous Aspergillus tracheobronchitis is the most severe form of IATB, and is characterized by extensive involvement of the tracheobronchial tree, a membranous layer covering the mucosal surface, and a poor prognosis, with mortality rates reportedly reaching up to $78 \%$ [8].

Recently, the following novel classification was proposed by Wu et al. [9] based on bronchoscopic findings: type I, superficial infiltration; type II, full-layer involvement; type III, occlusive (airway occlusion $\geq 50 \%$ ); and type IV, mixed. Methods to diagnose aspergillus infection are microscopic interpretation, antigen-based detection examination, molecular test, and culture, which is the gold standard for species-level recognition (demonstration). Species-level diagnosis can be safely made by correlating morphological characteristics in tissue sections with laboratory studies [10]. In the present case, bronchial biopsy was precluded due to severe thrombopenia. Instead of that, we decided to perform bronchoalveolar lavage (BAL) for cytological and microbiological examination. The microbiological examination of the BAL was positive for Aspergillus, and cytological examination was demonstrated cytological smear showing branching hyphae consistent with Aspergillus. Along with the clinical presentation, the severe

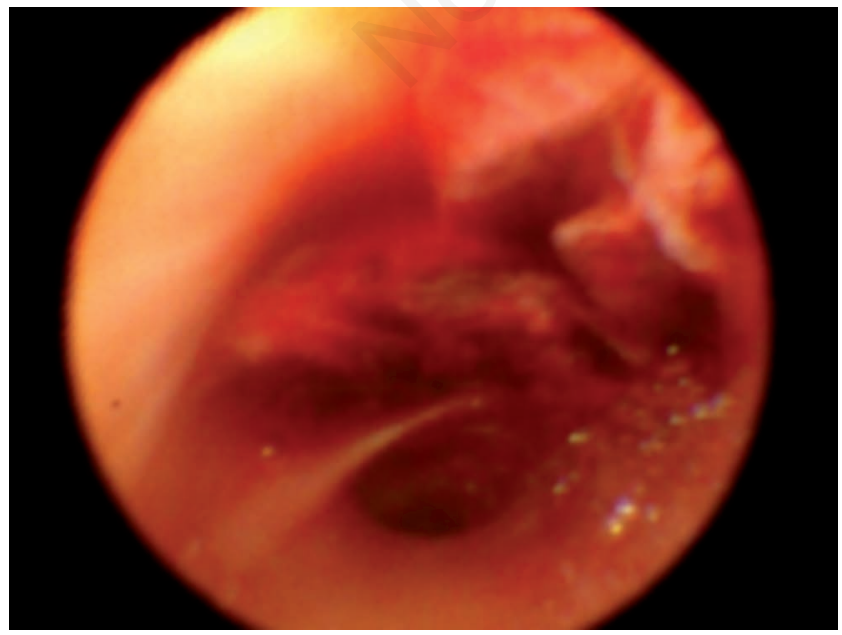

Figure 2. Bronchoscopic image showing obstruction of the right main lower lobe from thick mucus plugs with intense hemorrhagic appearance. underlying immunosuppression, CT features, and bronchoscopic findings all criteria are met for proven of IATB [11].

Relapse of hairy cell leukemia was considered the main predisposing factor for IATB in the present case. Previous reports suggest that although Aspergillus airway infection may complicate the course of various malignant diseases, the highest risk is associated with severely immunocompromised patients with hematological malignancies. Conversely, in patients with hematological malignancies and invasive Aspergillus infections, IATB is the less common form, and is responsible for $<10 \%$ of Aspergillus infections [8].

In the present case, concomitant malaria caused by Plasmodium falciparum could also be considered as an additional predisposing factor for IATB, because cases of invasive aspergillo-
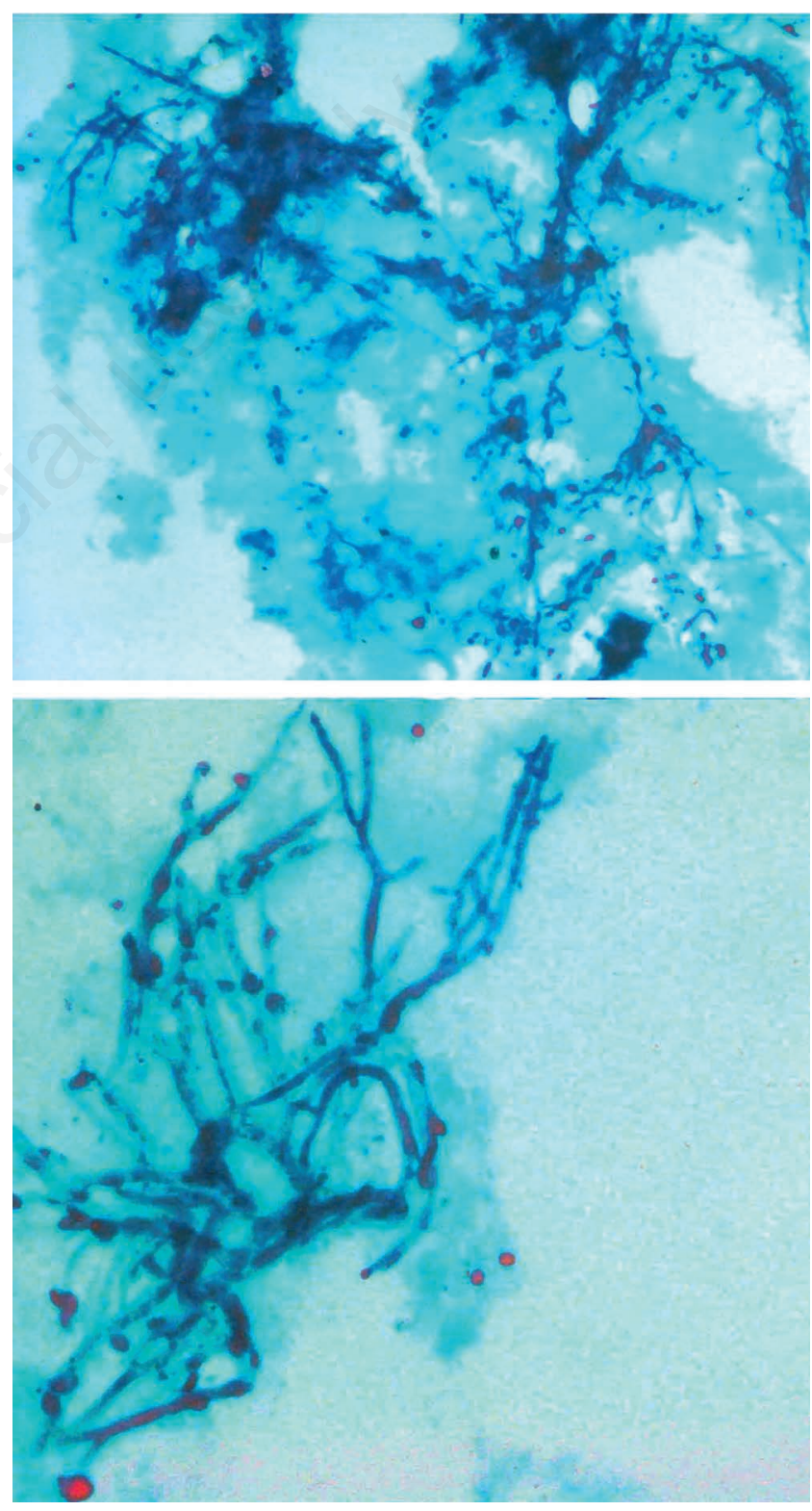

Figure 3. Cytological examination of bronchoalveolar lavage, showing hyaline, septate mycelium filaments $2-4 \mu \mathrm{m}$ in diameter with occasional branching at acute angles consistent with Aspergillus spp. Papanikolaou stain x 320. 
sis, pulmonary and tracheobronchial, complicating severe malaria caused by $P$. falciparum have previously been reported [12-16]. There is evidence that $P$. falciparum infection alters immune response by modulating both humoral and cell-mediated immunity [4]. Many components of cell-mediated immunity are reportedly affected by Plasmodium species, including dendritic cell activity, activation of $T$ cells by the dendritic cells, and macrophage function. Given the established role of macrophages and dendritic cells in optimal defense against Aspergillus species [5], an association between malaria and aspergillosis is apparent.

A biphasic dendritic cell response has been reported in animal models $[17,18]$. Early during infection or during non-lethal infection, dendritic cells are activated and mature dendritic cells are capable of inducing T cell proliferation [19]. During fatal infection or parasitemia however, dendritic cells are inhibited due to ingestion of increasing amounts of hemozoin, resulting in the induction of T-cell activation. In humans, Plasmodium-induced immunosuppression more commonly appears late in the acute phase of malaria and can persist for a long time after eradication of the parasites. These findings support our hypothesis that in the presenting patient, previous $P$. falciparum infection was an additional predisposing factor for the manifestation of Aspergillus infection, because the appearance of invasive aspergillosis occurred during the recovery phase of $P$. falciparum-induced malaria. To date, six cases of malaria-related invasive aspergillosis have been described [18]. The most common clinical manifestations were lung involvement (all cases), hemolysis, ARDS, cerebral malaria, and metabolic acidosis. The reported mortality rate is very high and mortality is mainly attributed to ARDS and cerebral dissemination. In the present case, lung involvement and associated ARDS was considered the primary cause of death.

Voriconazole is the primary choice of treatment for invasive aspergillosis. Liposomal amphotericin B and isavuconazole [20] are alternative options. Isavuconazole is a new extended-spectrum triazole with activity against Aspergillus, a safe profile, and excellent pharmacokinetics.

\section{Conclusions}

In conclusion, we present a rare case of obstructive Aspergillus invasive tracheobronchitis in a patient with hairy cell leukemia and previous Plasmodium falciparum infection. In patients who exhibit persistent high fever and unresponsiveness to antibiotics during the recovery phase of $P$. falciparum-induced malaria, the possibility of invasive aspergillosis or other fungal infections should be considered. Early diagnosis and rapid initiation of appropriate treatment may avert an unfavorable outcome in such cases.

\section{References}

1. Denning DW. Commentary: unusual manifestations of aspergillosis. Thorax 1995;50:812-3.

2. Fernández-Ruiz M, Silva JT, San-Juan R, et al. Aspergillus tracheobronchitis: report of 8 cases and review of the literature. Medicine (Baltimore) 2012 ;91:261-73.
3. Cho BH, Oh Y, Kang ES, Hong YJ, et al. Aspergillus tracheobronchitis in a mild immunocompromised. Host Tuberc Respir Dis 2014;77:223-6.

4. Wykes MN, Good MF. What really happens to dendritic cells during malaria? Nat Rev Microbiol 2008;6:864-70.

5. Cramer RA, Rivera A, Hohl TM. Immune responses against Aspergillus fumigatus: what have we learned? Curr Opin Infect Dis 2011;24:315-22.

6. Bassetti M, Peghin M, Vena A. Challenges and solution of invasive aspergillosis in non-neutropenic patients: a review. Infect Dis Ther 2018;7:17-27.

7. Shen Y, Zhang X, Lin W, et al. Liquid-based cytopathology test as a novel method to identify Aspergillus in patients with pulmonary aspergillosis. Sci Rep 2017;7:7528.

8. Tasci S, Glasmacher A, Lentini S, et al. Pseudomembranous and obstructive Aspergillus tracheobronchitis - optimal diagnostic strategy and outcome. Mycoses 2006;49:37-42.

9. Wu N, Huang Y, Li Q, et al. Isolated invasive Aspergillus tracheobronchitis: a clinical study of 19 cases. Clin Microbiol Infect 2010;16:689-95.

10. Kung VI, Chernock HD, Burnham. CAD Diagnostic accuracy of fungal identification in histopathology and cytopathology specimens. Eur J Clin Microbiol Infect Dis 2018;37:157-65.

11. De Pauw B, Walsh TJ, Donnelly JP, et al. Revised definitions of invasive fungal disease from the European Organization for Research and Treatment of Cancer/Invasive Fungal Infections Cooperative Group and the National Institute of Allergy and Infectious Diseases Mycoses Study Group (EORTC/MSG) Consensus Group. Clin Infect Dis 2008;46:1813-21.

12. Hocqueloux L, Bruneel F, Pages CL, Vachon F. Fatal invasive aspergillosis complicating severe Plasmodium falciparum malaria. Clin Infect Dis 2000;30:940-2.

13. Ruhnke M, Eichenauer E, Searle J, Lippek F. Fulminant tracheobronchial and pulmonary aspergillosis complicating imported Plasmodium falciparum malaria in an apparently immunocompetent woman. Clin Infect Dis 2000;30:938-40.

14. Wilson AP, Wright S, Bellingan G. Disseminated fungal infection following falciparum malaria. J Infect 2000;40:202-4.

15. Eckerle I, Ebinger D, Gotthardt D, et al. Invasive Aspergillus fumigatus infection after Plasmodium falciparum malaria in an immuno-competent host: case report and review of literature. Malar J 2009;8:167.

16. Däbritz J, Schneider M, Just-Nuebling G, Groll AH. Minireview: invasive fungal infection complicating acute Plasmodium falciparum malaria. Mycoses 2009;54:311-7.

17. Millington OR, Di Lorenzo C, Phillips RS, et al. Suppression of adaptive immunity to heterologous antigens during Plasmodium infection through hemozoininduced failure of dendritic-cell function. J Biol 2006;5:5.

18. Wykes MN, Liu XQ, Beattie L, et al. Plasmodium strain determines dendritic cell function essential for survival from malaria. PLoS Pathog 2007;3:e96.

19. Troye-Blomberg, M, Perlmann P. T cell functions in Plasmodium falciparum and other malarias. Prog Allergy 1988;41:253-87.

20. Maertens JA, Raad II, Marr KA, et al. Isavuconazole versus voriconazole for primary treatment of invasive mould disease caused by Aspergillus and other filamentous fungi (SECURE): a phase 3, randomised-controlled, non-inferiority trial. Lancet 2016;387:760-9. 